\title{
沙埋和种子大小对固沙禾草沙鞭的种子萌发 与幼苗出土的影响
}

\author{
朱雅娟 1,2 董 鸣 1 黄振英 ${ }^{*}$ \\ （1 中国科学院植物研究所植被数量生态学重点实验室, 北京 100093）（2 中国科学院研究生院, 北京 100039）
}

摘 要 该文研究了野外条件下不同深度的沙埋对沙鞭( Psammochloa villosa) 种子萌发和幼苗出土的影响, 以及温 室条件下种子大小对不同深度沙埋后的种子萌发和幼苗出土的影响。结果表明,沙埋深度显著影响沙鞭的种子萌 发率、幼苗出土率和种子休眠率。沙子表面的种子不能萌发。 $2 \mathrm{~cm}$ 的浅层沙埋时的种子萌发率和幼苗出土率最 高, $1 \mathrm{~cm}$ 沙埋的种子萌发率和幼苗出土率次之。沙埋深度超过 $2 \mathrm{~cm}$ 之后, 沙鞭的种子萌发率和幼苗出土率与沙埋 深度呈负相关。 $2 \mathrm{~cm}$ 的种子休眠率最低。从 $2 \sim 12 \mathrm{~cm}$, 种子休眠率随着沙埋深度的增加而增加。在幼苗能够出土 的深度 $(1 \sim 6 \mathrm{~cm})$, 幼苗首次出土所需的时间随着沙埋深度的增加而延长。种子大小对沙鞭的种子萌发率没有显著 影响。但是在深层沙埋 $(6 \mathrm{~cm})$ 时, 与小种子相比, 大种子产生的幼苗的出土率较高。从 $2 \sim 6 \mathrm{~cm}$, 大种子形成的幼苗 的茎长度都较长。

关键词 沙鞭 沙埋深度 种子大小 种子萌发 幼苗出土 种子生态学

\section{EFFECTS OF SAND BURIAL AND SEED SIZE ON SEED GERMINATION AND SEEDLING EMERGENCE OF PSAMMOCHLOA VILLOSA}

\author{
ZHU Ya-Juan ${ }^{1,2}$ DONG Ming ${ }^{1}$ and HUANG Zhen-Ying ${ }^{1}{ }^{*}$ \\ (1 Laboratory of Quantitative Vegetation Ecology, Institute of Botany, Chinese Academy of Sciences, Beijing 100093, China) \\ (2 Graduate School of Chinese Academy of Sciences, Beijing 100039, China)
}

\begin{abstract}
Sand burial is a common phenomenon in sand dune ecosystems all over the world. Although many studies concerning the effects of sand burial on seed germination and seedling emergence have been conducted in many parts of the world, such studies are rare in China. In the summer of 2003 , we studied the effects of sand burial depth $(0,1,2,4,6,8,10$ and $12 \mathrm{~cm})$ and seed size on seed germination and seedling emergence of Psammochloa villosa, an important psammophyte that establishes on mobile sand dunes in deserts and sandy lands of North China. Seeds were collected in their natural habitat in Mu-Us Sandland, Ordos Plateau, in 2002 and stored at $-18{ }^{\circ} \mathrm{C}$. The average seed mass of $P$. villosa was $(5.51 \pm 0.05) \mathrm{mg}$. Seeds were sorted into 3 groups based on their mass: small $(4-4.9 \mathrm{mg}$, mean $=(4.489 \pm 0.012) \mathrm{mg})$, medium $(5-$ $5.9 \mathrm{mg}$, mean $=(5.457 \pm 0.012) \mathrm{mg})$, and large $(6-6.9 \mathrm{mg}$, mean $=(6.415 \pm 0.011) \mathrm{mg}) . \mathrm{We}$ conducted two experiments, one in the field and the other in a non-heated greenhouse. In the second experiment, burial depth was $1,2,4,6$ and $8 \mathrm{~cm}$. Before burial experiments, all seeds were treated with cold stratification in $5{ }^{\circ} \mathrm{C}$ for 4 weeks in order to break dormancy.

We found that sand burial depth had significant effects on seed germination and seedling emergence of $P$. villosa. Experiments revealed that the highest percentage of seeds that germinated and seedlings that emerged occurred at $2 \mathrm{~cm}$ burial depth. Few seeds lying on the surface of the sand germinated, and, those that did germinate, could not anchor their roots into the soil and seedlings did not establish successfully. The next greatest germination and emergence percentage occurred at the $1 \mathrm{~cm}$ burial depth. The timing of emergence was delayed with deeper burial depth. As burial depth increased from 2 to $12 \mathrm{~cm}$, the percentage of seeds that germinated and emerged declined, a higher number of seeds remained dormant, and the number of days before first emergence increased. As sand burial depth increased, some seeds were able to germinate but the seedlings were not
\end{abstract}


able to emerge possibly because the seeds did not have enough energy. Seedlings that could not emerge, etiolated, died and decomposed in the sand. At all depths, the seeds that could not germinate remained dormant. Possible reasons for sustained dormancy include poor aeration, high sand moisture, low soil temperature, poor $\mathrm{O}_{2}$ content, higher $\mathrm{CO}_{2}$ levels in soil and low light intensity. The dormant seeds, which form soil seed banks, are very important for the long-term survival of this species. The dormant seeds can germinate when sand erosion decreases the burial depth and the seeds are exposed to optimal environmental conditions, such as sand depth. However, our experiment revealed that seeds buried at deeper depths had higher mortality rates ( 15 $35 \%)$. The high mortality might be due to fungal infection, water-soluble germination inhibitors in the seed coat, and an unfavorable microenvironment and low $\mathrm{O}_{2}$ concentrations.

Seed size did not affect the percentage of seeds that germinated or remained dormant, but had a remarkable effect on the percentage of seedlings that emerged at different sand burial depths. At greater depths ( $>6$ $\mathrm{cm}$ ), large seeds had higher rates of seedling emergence than medium and small seeds. From 1 to $6 \mathrm{~cm}$ depth, the seedling shoots that originated from large seeds were longer than those from small seeds; also, at 2 to $6 \mathrm{~cm}$ depth, seedling shoots that originated from medium sized seeds were longer than those from small seeds. Therefore, we suggest larger seeds have ecological advantages in sand dune ecosystems. Larger seeds have a larger endosperm, contain more energy, and thus can emerge from deeper burial depths. This will benefit seedling growth in early stages and help them to successfully establish under extreme desert conditions. Sand burial might be a selective pressure on seed size; therefore, seed polymorphism as an evolutionary strategy can benefit the renewal of $P$. villosa populations. In sand dune habitats, $P$. villosa produced different sizes of seeds that could emerge from different depths of sand burial. The diversity of seed size could enhance opportunities for seedling establishment in this heterogeneous environment allowing plants to respond to different sand burial depths, enhance their ability to adapt to the changing environment, and increase survival.

Key words Psammochloa villosa, Sand burial depth, Seed size, Seed germination, Seedling emergence,

Seed ecology

在荒漠地区, 流动沙丘是植物生长发育最严酷 的生境之一(Danin, 1996)。生长在流动沙丘上的植 物、种子和幼苗经常会遭受不同程度的沙埋(Maun, 1981，1998）。这可能是影响植物在沙丘生境中建成 的一个重要因素(van der Valk, 1974)。

沙埋对种子大小、种子萌发、幼苗出土、幼苗定 居以及成年植物的进化有很强的选择压力 (Danin, 1996; Maun, 1998)。它使种子得到一定的保护, 例 如避开土壤表面的各种采食者(包括鸟类、昆虫和啮 齿动物等), 避免沙土表面的高温和干燥的威胁等。 在一定的深度范围内, 沙埋可以促进种子萌发、幼苗 出土和幼苗生长, 因为沙埋改善了植物的微环境, 例 如温度下降、土壤含水量增加等。但是过量的沙埋 会抑制种子萌发和幼苗出土, 因为沙层深处通气不 良、缺乏光照并且缺少温度变化 ( van Assche \& Vanlerberghe, 1989; Vleeshouwers, 1997)。一部分种子会 受环境因素的影响而进入强迫休眠, 形成土壤种子 库, 从而有利于物种的长期生存 (Maun, 1998)。

般来说, 种子的质量越大, 所含的能量越多, 形成的 幼苗有能力穿透更深的沙层。这种趋势在植物的种 内和种间都存在 (Maun, 1981; Yanful \& Maun, 1996a）。

植物只有使它们的种子能够从一定深度的沙埋
中萌发和长出幼苗( Maun \& Lapierre, 1984; Zhang \& Maun, 1990b, 1990c; Huang \& Gutterman, 1998; 黄振 英等, 2001 ), 并且在幼苗阶段忍耐一定程度的沙埋 (Harris \& Davy, 1987; Sykes \& Wilson, 1990; Yanful \& Maun, 1996b; Greipsson \& Davy, 1996; 张称意等, 2002), 才能成功地在经常发生沙埋的流动沙丘上 定居。这是生长在流动沙丘上的植物特有的重要的 生理生态特征之一。

沙鞭( Psammochloa villosa) 是禾本科沙鞭属的多 年生草本植物, 具有发达的根茎, 是一种优良的固沙 植物。它主要分布在我国北方的荒漠地区和蒙古国 境内 (刘瑛心，1985)。作为对流动沙丘上的干旱、 沙埋等特殊环境胁迫的适应, 沙鞭具有典型的旱生 沙生特征, 是沙地植物群落的优势种 (马毓泉, 1994)。在鄂尔多斯高原的毛乌素沙地中, 沙鞭主要 生长在流动沙丘、半流动沙丘以及沙地半灌木从中。 沙鞭是一种克隆植物, 能够通过根状茎进行快速的 无性繁殖。这是它对流动沙丘的一种特殊的生态适 应。沙鞭的克隆生长与克隆整合特性对它在水分短 缺、营养贫乏、生境破碎以及和经常遭受扰动的沙地 生境中的生存有重要贡献 (董鸣, 1999; Dong \& Alaten, 1999); 而且由于它具有较大的地下生物量 配置, 其固沙能力很强( Roels et al ., 2001)。 
根据我们多年的野外观察, 在毛乌素沙地中, 当 年降雨量达到 350 400 mm 时, 沙鞭就能够通过有 性繁殖产生大量的种子。但是在它的自然生境中, 植株个体大多数都是由克隆生长产生的分株, 由种 子萌发形成的幼苗却比较少。对于一个植物种群而 言, 有性生殖比无性生殖更重要, 因为有性生殖过程 能够产生具有新的适应特征的基因型, 从而增加植 物的生存和竞争能力。因此有必要探讨沙鞭的种子 萌发和幼苗出土这一植物生活史中的关键阶段, 深 入研究沙鞭的种子萌发生态学。

能够在荒漠条件下生存和发育的植物都有其特 殊的种子萌发和幼苗发育机制(Gutterman，1993)。 因此我们推测, 沙鞭种子也有特殊的萌发策略来适 应严酷的沙丘环境。以前的实验表明, 新成熟的沙 鞭种子具有非深度生理休眠, 是对其生境中冬季低 温的一种适应(黄振英, 2003)。本文是沙鞭种子萌 发生态学研究的一部分, 主要报道了沙埋对沙鞭种 子萌发和幼苗出土的影响, 以及沙鞭种子萌发对于 这一环境胁迫的适应策略, 以期为沙化草地的人工 治理和受损生态系统的重建提供理论依据。

\section{1 材料和方法}

沙鞭的成熟颖果 (以下简称种子)于 2002 年 10 $\sim 11$ 月期间采自中国科学院植物研究所内蒙古鄂 尔多斯沙地草地生态研究站附近自然分布的沙鞭植 株上。该站 $\left(39^{\circ} 02^{\prime} \mathrm{N} ; 109^{\circ} 51^{\prime} \mathrm{E}\right)$ 位于内蒙古自治区 鄂尔多斯高原南部, 海拔 $1355 \mathrm{~m}$, 年平均气温为 6.0 $\sim 8.5{ }^{\circ} \mathrm{C}$, 平均年降雨量为 $358.3 \mathrm{~mm}$, 并且集中在 $6 \sim 9$ 月。

待包裹成熟颖果的颖壳自然脱落后, 将清理干 净的颖果放在布袋中败藏在 $-18{ }^{\circ} \mathrm{C}$ 下备用( International Seed Testing Association, 1985)。在实验开始之 前对种子进行 4 星期的低温 $\left(5{ }^{\circ} \mathrm{C}\right)$ 层积处理以打破 内生休眠 (黄振英, 2003)。

\section{1 种子的大小和质量}

实验开始之前, 测定种子的大小和质量。测定 1000 粒种子的质量, 4 个重复, 结果以平均值 \pm 标 准误差表达。随机取 300 粒种子, 用电子天平逐个 测量其质量, 分析不同质量的沙鞭种子的所占的比 例。用最小刻度为 $0.5 \mathrm{~mm}$ 的钢尺测定 20 粒种子的 直径和长度, 结果均为估测的平均值。

1.2 沙埋深度对种子萌发和幼苗出土的影响

实验于 2003 年 8 月 4 日在生态站沙鞭的自然 生境附近的露天实验场进行。实验中所用的种子质
量为 $(5.5 \pm 0.5) \mathrm{mg}$ 左右。经过 4 星期 $5^{\circ} \mathrm{C}$ 的低温 层积处理后, 这些种子已经打破了内生休眠, 在 20 $\sim 30^{\circ} \mathrm{C}$ 温度内, 其萌发率可达 $100 \%$ 。沙埋深度分 别为: 0 (对照) 、 $1 、 2 、 4 、 6 、 8 、 10$ 和 $12 \mathrm{~cm}$, 共 8 个处理。 每个处理 8 个重复, 每个重复是将 50 粒质量为 5.5 $\mathrm{mg}$ 左右的种子均匀摆放在直径 $10 \mathrm{~cm}$ 、高 $12 \mathrm{~cm}$ 、装 有 $5 \mathrm{~cm}$ 厚沙子的塑料花盆的表面, 再分别进行不同 深度的沙埋处理。所用的沙子取自沙鞭自然生境中 的沙丘上, 用尼龙网过滤除去杂质。将 72 个花盆随 机埋在一个长 $\times$ 宽 $\times$ 高分别为 $1 \mathrm{~m} \times 3.5 \mathrm{~m} \times 0.25 \mathrm{~m}$ 的实验场地内, 花盆内外的沙子保持同样的水平。 每天分别向每个花盆加入等量的地下水, 以保持沙 土湿润。当白天温度超过 $30{ }^{\circ} \mathrm{C}$ 时用遮阳网遮荫。 沙层表面的出苗以胚芽露出为标准。对出苗的检测 和记录共进行 $30 \mathrm{~d}$ 。实验开始 2 周内每天 $8: 00$ 和 14:00 分别记录各沙埋深度的温度, 随机测 3 个值, 取平均值。实验期间各沙埋深度的土壤温度在 15 $\sim 32{ }^{\circ} \mathrm{C}$ 之间变化。从 $0 \sim 12 \mathrm{~cm}$, 随着沙埋深度的增 加, 温差逐渐降低。

实验结束后, 将花盆里的沙子用尼龙网过滤, 找 出未萌发的种子、被真菌感染而死亡的种子以及萌 发但未出苗的种子。将每个花盆里未萌发的种子带 回实验室, 分别放在直径 $90 \mathrm{~mm}$, 垫有两层滤纸的培 养血中, 加入 $2.5 \mathrm{ml}$ 蒸馏水, 在 $25{ }^{\circ} \mathrm{C}$ 、光照下进行萌 发, 以检测未萌发种子的活力。种子萌发以胚根露 出为标准。种子死亡以被真菌感染而且没有萌发为 标准。种子萌发过程中每 $24 \mathrm{~h}$ 检测 1 次, 并将已经 萌发的种子移走。检测共进行 $20 \mathrm{~d}$ 。分别统计萌发 的种子 (即沙埋时休眠的种子) 和死种子的数量, 并 计算种子休眠率和死亡率 ( 包括沙埋之后死亡的 种子和检测活力时死亡的种子)。

1.3 种子大小与沙埋深度对种子萌发和幼苗出土 的影响

实验在无热源温室中进行。沙埋深度分别为 0 (对照)、1、2、4、6 和 $8 \mathrm{~cm}$ 。用万分之一电子天平对 单个种子称重并将种子按照质量分为 3 组: 小 $(4.0$ $\sim 4.9 \mathrm{mg}$, 平均为 $(4.489 \pm 0.012) \mathrm{mg}$ 、中 $(5.0 \sim 5.9$ $\mathrm{mg}$, 平均为 $(5.457 \pm 0.012) \mathrm{mg}$ ) 和大 $(6.0 \sim 6.9 \mathrm{mg}$, 平均为 $(6.415 \pm 0.011) \mathrm{mg}$, 每组 600 粒种子。将 25 粒种子按照不同的深度种在直径 $15 \mathrm{~cm}$ 、高 $15 \mathrm{~cm}$ 的塑料花盆中。每个处理(同一深度同一质量) 4 个 重复, 共 72 盆。盆底的排水孔用小石子堵住以防止 沙子流失。沙子用篮子除去杂物并用自来水洗过 1 次。先加入一定量的沙子, 加适量自来水使沙子表 
面湿润, 将种子均匀地平放在沙子表面, 再加沙子到 要求的深度。浇足量自来水使沙子湿润, 以后每天 早晨 8:00 和下午 14:00 各浇 1 次水。每天检测幼 苗出土的情况。记录温室里的温度和湿度的变化范 围。实验期间温室里的气温在 $19 \sim 36{ }^{\circ} \mathrm{C}$ 之间变化, 相对湿度在 24\% 65\%之间变化。实验从 2004 年 3 月 6 日开始, 到 4 月 5 日结束, 共进行 $30 \mathrm{~d}$ 。

实验结束后, 分别在每个花盆中随机选择 10 株 幼苗, 测量茎的长度, 分析沙埋对幼苗生长的影响。 用 20 目的土壤篮过滤每个花盆中的沙子, 找出未萌 发的种子和萌发但未出苗的种子。在室温下用 $1 \%$ 的 TTC 溶液检测未萌发种子的活力。将种子剖开, 放在直径 $50 \mathrm{~mm}$ 的培养血中, 加入 $2 \mathrm{ml} \mathrm{TTC}$ 溶液, 24 $\mathrm{h}$ 之后, 胚呈红色的是活种子, 即沙埋时休眠的种 子。

\section{4 数据分析}

实验结果以百分率 \pm 标准误差表达, 或者根据 实验要求检验种子萌发或幼苗出土的差异程度。如 果实验结束之后某个处理中的种子没有萌发但保持 活力,幼苗首次出土的时间按 $30 \mathrm{~d}$ 计算。通过单因 子方差分析 (One-way ANOVA) 或双因子方差分析 (Two-way ANOVA) 检验差异的显著性; 如果差异显 著,再利用 Tukey's 检验确定平均值之间的差异性 (Sokal \& Rohlf, 1981)。

\section{2 实验结果}

\section{1 种子的大小和质量}

沙鞭种子为深褐色, 呈长纺锤形, 直径约 1.0 $1.5 \mathrm{~mm}$, 长度是 $5.0 \sim 7.0 \mathrm{~mm}$; 种子平均质量为 $(5.51 \pm 0.05) \mathrm{mg}$ 。质量 $\leqslant 4.0,4.1 \sim 5.0,5.1 \sim$ $6.0,6.1 \sim 7.0$ 以及 $>7.0 \mathrm{mg}$ 的种子分别占 $5.3 \%$ 、 $26 \% 、 44.3 \% 、 20.3 \%$ 和 $4.1 \%$ 。

\section{2 沙埋深度对种子萌发和幼苗出土的影响}

沙鞭的幼苗出土率在不同的沙埋深度表现出显 著差异 $(p<0.001)$ (图 1, 图 2)。沙埋 $1 \sim 2 \mathrm{~cm}$ 的幼 苗出土率最高。从 $2 \sim 8 \mathrm{~cm}$, 随着沙埋深度增加, 幼 苗出土率显著降低。从 $8 \sim 12 \mathrm{~cm}$, 能够出土的幼苗 只有 $1 \%$ 左右, 差异不显著。

沙埋深度对沙鞭的种子萌发率有显著影响 ( $p$ $<0.001$ ) (图 2)。从 $0 \sim 2 \mathrm{~cm}$, 随着沙埋深度的增加, 沙鞭的种子萌发率显著增加。沙埋 $2 \mathrm{~cm}$ 处的种子 萌发率最高。从 $2 \sim 6 \mathrm{~cm}$, 随着沙埋深度增加, 种子 萌发率显著降低。从 $6 \sim 12 \mathrm{~cm}$, 种子萌发率都低于 $10 \%$, 差异不显著(图 2)。
图 1 不同沙埋深度的沙鞭种子在 $30 \mathrm{~d}$ 内的幼苗 出土率 (平均值 \pm 标准误)

Fig. 1 Seedling emergence (Mean $\pm S E$ ) of Psammochloa villosa's seeds buried in different depths in 30 days

$8 、 10$ 和 $12 \mathrm{~cm}$ 的幼苗出土率为 0 Emergence percentage of 8,10 and $12 \mathrm{~cm}$ is 0

经过检测未萌发种子的生理状态表明, 部分种 子进入休眠状态。在沙层表面, 因为胚暴露在空气 中以及强烈的蒸发造成的水分缺乏而导致 $30 \%$ $40 \%$ 的种子进入强迫休眠状态。在沙层下, 部分种 子因为沙埋而进入强迫休眠状态。从 $2 \sim 6 \mathrm{~cm}$, 种子 休眠率随着沙埋深度的增加而显著增加 ( $p<$ 0.001 )。从 6 12 cm, 种子休眠率保持在 $60 \%$ 左右 (图 2)。另一部分没有萌发的种子已经死亡。沙鞭 种子在沙层表面的死亡率最高, 为 $43 \%$ 左右; $1 \sim 2$ $\mathrm{cm}$ 处的种子死亡率最低, 为 $15 \%$ 左右; $4 \sim 12 \mathrm{~cm}$ 的 种子死亡率为 30\% 35\% (图 2)。

沙埋对幼苗首次出土所需的时间也有显著影响 $(p<0.001)$ 。沙埋深度为 $2 \mathrm{~cm}$ 时幼苗出土所需的 时间最短, 大约是 $8 \mathrm{~d}$ 。种子在沙层表面时, 幼苗出 土(子叶露出)所需的时间显著大于 $1 \sim 2 \mathrm{~cm}$ 沙埋时 幼苗出土所需的时间。从 $2 \sim 6 \mathrm{~cm}$, 沙埋越深, 幼苗 出土所需的时间越长 (图 3)。

2.3 种子大小和沙埋深度对种子萌发和幼苗出土 的影响

种子大小对幼苗出土率在总体上有显著影响。 $1 \sim 2 \mathrm{~cm}$ 的浅层沙埋时的幼苗出土率最高。从 $2 \sim 6$ $\mathrm{cm}$, 随着沙埋深度增加, 幼苗出土率降低。沙埋深 度超过 $8 \mathrm{~cm}$ 之后, 只有少量幼苗能够长出土壤表面 (图 4)。在 1 4 cm 的浅层沙埋时, 种子大小对幼苗 出土率没有显著影响, 大种子没有明显的优势。但 是当沙埋深度加大到 $6 \mathrm{~cm}$ 时, 种子大小对幼苗出土 率的影响达到显著水平 $(p=0.007)$, 中等大小的 
图 2 沙埋深度对沙鞭的种子萌发率 $(S G$ )、幼苗出土率 $(S E)$ 、种子休眠率 $(S D$ o $)$ 和种子死亡率 $(S D \mathrm{e})$ (平均值 \pm 标准误) 的影响

Fig.2 Effects of sand burial on the percentage of seed germination $(S G)$, seedling emergence $(S E)$, seed dormant $\left(S D_{0}\right)$ and seed death $(S D e)($ Mean $\pm S E$ ) of Psmmochloa villosa

根据 Tukey's 检验, 各组之内由不同小写字母标记的值之间的差异是显著的 $(p<0.05)$ In each group, the values followed by different letters in lower case are significantly different $(p<0.05)$, according to Tukey's test

图 3 不同深度的沙埋对沙鞭幼苗首次 出土所需时间 (平均值 \pm 标准误)的影响

Fig.3 Effects of sand burial on days of first emergence (Mean $\pm S E$ ) of Psammochloa villosa seedling 图注同图 2 The note see Fig.2

种子和大种子的幼苗出土率比小种子高。沙埋深度 和种子大小的相互作用对幼苗出土率的影响不显著 (表 1)。

种子大小在总体上对种子萌发率没有影响。在 各个沙埋深度, 不同大小的种子的萌发率都没有显
图 4 沙埋深度和种子大小对沙鞭的幼苗出土率 (平均值 \pm 标准误) 的影响

Fig. 4 Effects of sand burial depth and seed size on percentages of seedling emergence (Mean $\pm S E$ ) of Psammochloa villosa 图注同图 2 The note see Fig. 2

著差异。沙土表面的种子不能萌发。1 4 cm 的种 子萌发率最高。从 $4 \sim 8 \mathrm{~cm}$, 随着沙埋深度增加, 种 子萌发率降低。 $8 \mathrm{~cm}$ 处仍然有大约 $33 \% \sim 38 \%$ 的 种子可以萌发 (图 5)。但是在 4 周的实验期间, 大 部分萌发的种子却不能长出沙土表面, 在土 壤里被真菌感染而死亡。在各个沙埋深度, 除 了 $4 \mathrm{~cm}$ 处的大种子的萌发率显著高于小种子之外 
表 1 沙埋深度和种子大小以及二者的相互作用对沙鞭的幼苗出土率、种子萌发率和种子休眠率的影响的双因素方差分析

Table 1 Two-way ANOVA analysis of effects of burial depth, seed size and their interaction on the percentage of seedling emergence, seed germination and seed dormancy of Psammochloa villosa

\begin{tabular}{|c|c|c|c|c|c|}
\hline $\begin{array}{c}\text { 变差来源 } \\
\text { Source of variation }\end{array}$ & $\begin{array}{c}\text { 自由度 } \\
d f\end{array}$ & $\begin{array}{c}\text { 平方和 } \\
S S\end{array}$ & $\begin{array}{c}\text { 均方 } \\
M S\end{array}$ & $\begin{array}{c}F \text { 值 } \\
F \text {-value }\end{array}$ & $\begin{array}{c}p \text { 值 } \\
p \text {-value }\end{array}$ \\
\hline \multicolumn{6}{|c|}{ 幼苗出土率 Seedling emergence (\%) } \\
\hline 沙埋深度 Burial depth（cm） & 5 & 10.945 & 2.189 & 178.339 & $<0.001$ \\
\hline 种子大小 Seed size & 2 & 0.132 & 0.066 & 5.369 & 0.007 \\
\hline 深度 $\times$ 大小 Depth $\times$ Size & 10 & 0.159 & 0.016 & 1.298 & 0.255 \\
\hline 误差 Error & 54 & 0.663 & 0.012 & & \\
\hline 总和 Total & 71 & 11.988 & & & \\
\hline \multicolumn{6}{|c|}{ 种子萌发率 Seed germination (\%) } \\
\hline 沙埋深度 Burial depth（cm） & 5 & 4.626 & 0.925 & 870.736 & $<0.001$ \\
\hline 种子大小 Seed size & 2 & 0.009 & 0.005 & 4.421 & 0.017 \\
\hline 深度 $\times$ 大小 Depth $\times$ Size & 10 & 0.012 & 0.001 & 1.147 & 0.347 \\
\hline 误差 Error & 54 & 0.057 & 0.001 & & \\
\hline 总和 Total & 71 & 4.705 & & & \\
\hline \multicolumn{6}{|c|}{ 种子休眠率 Seed dormancy（\%） } \\
\hline 沙埋深度 Burial depth（cm） & 5 & 7.217 & 1.443 & 616.618 & $<0.001$ \\
\hline 种子大小 Seed size & 2 & 0.016 & 0.008 & 3.456 & 0.039 \\
\hline 深度 $\times$ 大小 Depth $\times$ Size & 10 & 0.013 & 0.001 & 0.573 & 0.828 \\
\hline 误差 Error & 54 & 0.126 & 0.002 & & \\
\hline 总和 Total & 71 & 7.372 & & & \\
\hline \multicolumn{6}{|c|}{ 幼苗的茎长度 Seedling length $(\mathrm{cm})$} \\
\hline 沙埋深度 Burial depth（cm） & 3 & 4295.757 & 1431.9 & 392.245 & $<0.001$ \\
\hline 种子大小 Seed size & 2 & 164.210 & 82.105 & 22.491 & $<0.001$ \\
\hline 深度 $\times$ 大小 Depth $\times$ Size & 6 & 131.288 & 21.881 & 5.994 & $<0.001$ \\
\hline 误差 Error & 423 & 1544.184 & 3.651 & & \\
\hline 总和 Total & 434 & 6135.449 & & & \\
\hline
\end{tabular}

种子大小对种子休眠率也没有影响。沙土表面 的种子都处于休眠状态。1 2 cm 的种子休眠率最 低。从 $2 \sim 8 \mathrm{~cm}$, 随着沙埋深度增加, 种子休眠率提 高。6 和 $8 \mathrm{~cm}$ 处分别有大约 $30 \% \sim 33 \%$ 和 $48 \% \sim$ $52 \%$ 的种子处于休眠状态 (图 6)。在各个沙埋深 度, 除了 $4 \mathrm{~cm}$ 处的小种子的休眠率显著高于大种子 之外 ( $p=0.039)$, 其它各个深度的不同大小的种 子的休眠率差异不显著。沙埋深度和种子大小的相 互作用对种子休眠率的影响不显著(表 1)。

种子大小对幼苗的茎的长度有显著的影响 ( $p$ $<0.001)$ 。随着沙埋深度的增加, 茎的长度也增加。

图 5 沙埋深度和种子大小对沙鞭的种子萌发率 (平均值 \pm 标准误) 的影响

Fig.5 Effects of sand burial depth and seed size on percentages of seed germination (Mean $\pm S E$ ) of Psammochloa villosa 图注同图 2 The note see Fig.2

( $p=0.017)$, 其它深度的不同大小的种子的萌发率 差异不显著。沙埋深度和种子大小的相互作用对种 子萌发率的影响不显著(表 1 )。
据观察, 虽然在 $1 \sim 2 \mathrm{~cm}$ 的浅层沙埋时, 幼苗的茎比 较短, 但是它们却长得比较粗壮一些。从 $1 \sim 6 \mathrm{~cm}$, 在每个沙埋深度, 大种子形成的幼苗长度显著大于 小种子形成的幼苗长度; 从 $2 \sim 6 \mathrm{~cm}$, 在每个沙埋深 度, 中等大小的种子形成的幼苗长度显著大于小种 子形成的幼苗长度 (图 7)。种子大小和沙埋深度的 相互作用对幼苗的茎的总长度和地面上的茎长度的 影响达到了显著水平 $(p<0.001)$ (表 1)。 
图 6 沙埋深度和种子大小对沙鞭的种子休眠率 (平均值 \pm 标准误) 的影响

Fig.6 Effects of sand burial depth and seed size on percentages of seed dormancy (Mean $\pm S E$ ) of Psammochloa villosa 图注同图 2 The note see Fig.2
图 7 沙埋深度和种子大小对沙鞭的幼苗的 茎长度 (平均值 \pm 标准误) 的影响

Fig.7 Effects of sand burial depth and seed size on seedling length (Mean $\pm S E$ ) of Psammochloa villosa 图注同图 2 The note see Fig.2

\section{3 讨 论}

在沙丘生境中, 植物的种子在被传播之后, 除了 一部分会被各种食果动物 (包括昆虫、鸟类和啮齿类 等)消耗掉之外,一些种子会由于雨水、风、动物活 动、洪水、调落物分解以及自身结构等因素而被埋在 浅层土壤里, 然后随着沙子运动再被埋到不同的深 度(Maun，1998)。种子被沙埋后可能有 4 种命运: 1) 种子萌发而且幼苗出土; 2) 种子萌发了但幼苗没 有出土, 沙层中的幼苗可能会受到真菌侵染而分解; 3) 种子没有萌发并且被其它生物 (例如啮齿类和真 菌)破坏;4)种子进入强迫休眠或诱导休眠, 成为土
壤种子库的一部分 (Maun, 1998)。沙埋后种子的萌 发将受到温度、光照、土壤湿度和盐分等综合因素的 影响(Huang \& Gutterman, 1998; 黄振英和 Gutterman, 2000)。

野外实验结果表明, 沙埋深度对沙鞭的种子萌 发有显著的影响。高温与强烈的水分蒸发抑制了沙 层表面的种子的萌发。观察表明,即使沙子表面的 种子萌发了, 它们的根系也很难扎入沙层中, 幼苗无 法成功定居。从 $2 \sim 12 \mathrm{~cm}$, 种子被埋藏的越深, 种子 萌发率就越低, 种子萌发率与沙埋深度呈负相关。 前人在对一些海滨沙丘植物 (Maun, 1981; Maun \& Lapierre, 1986; Zhang \& Maun, 1990a; Chen \& Maun, 1999)进行了种子萌发生态学的研究后也得出了种 子萌发率与沙埋深度呈负相关的结论。许多因素能 够影响种子在沙层中的萌发。3 种蒿属 (Artemisia) 的荒漠沙丘植物的种子在不同沙层深度的萌发是光 照、土壤水分和土壤温度等因素综合作用的结果; $0.5 \sim 2 \mathrm{~cm}$ 是适宜的沙埋深度 (Huang \& Gutterman, 1998; 黄振英和 Gutterman, 2000; 黄振英等, 2001)。 结合前人的观点和我们的实验结果, 我们认为沙鞭 种子萌发需要 $1 \sim 2 \mathrm{~cm}$ 的浅层沙埋, 因为适量的沙 层能够增加种子周围的土壤的紧实度和相对湿度, 从而提高萌发的机会。

沙埋深度对幼苗出土的影响比对种子萌发的影 响更大(Maun, 1998; Baskin \& Baskin, 1998)。研究 表明, 沙埋超过一定的深度之后,一些植物的种子仍 然可以萌发, 但是它们的幼苗却不能出土。例如, 冰 草属的 Agropyron psammophilium 的种子在 $2 \sim 12 \mathrm{~cm}$ 的萌发率都可以达到 $90 \%$ 以上, 但是深度超过 $8 \mathrm{~cm}$ 之后幼苗不能出土 (Zhang \& Maun, 1990c); Ammorphila breviligulata, Cackie edentula, Corispermum hysopifolium 以及披碱草属的 Elymus canadensis 的种子在 0 $\sim 12 \mathrm{~cm}$ 都可以萌发, 但它们的幼苗能够出土的深 度分别只有 $6 、 10 、 8$ 和 $12 \mathrm{~cm}$ (Maun \& Lapierre, 1986); Calamovilfa longifolia 的种子在 $1 \sim 12 \mathrm{~cm}$ 都可 以萌发, 但其幼苗能够出土的最大深度仅为 $8 \mathrm{~cm}$ (Maun, 1981)。过量的沙埋也降低了幼苗的出土率 和出土速度 (Zhang \& Maun, 1990b)。沙鞭幼苗的最 大出土率出现在 $2 \mathrm{~cm}$ 的浅层沙埋时。从 $2 \sim 6 \mathrm{~cm}$, 沙埋深度越深, 幼苗出土率越低。沙埋超过 $6 \mathrm{~cm}$ 后, 仅有个别幼苗能够出土。种子萌发后, 幼苗能否 出土取决于种子的胚乳中所含的能量和沙埋的深度 （Maun, 1998；Baskin \& Baskin, 1998）。沙鞭的一些 种子萌发后, 幼苗不能长出沙层表面。这可能是因 
为沙层太深, 种子没有足够的能量使幼苗出土。观 察表明, 不能出土的幼苗表现出明显的黄化现象, 这 可能是由于沙层中光照不足造成的。这些幼苗可能 会在沙层中死亡并腐烂。在幼苗能够出土的深度 (1 $\sim 6 \mathrm{~cm}$ ), 沙埋深度越深, 幼苗首次出土所需的时间 越长。

沙鞭的种子休眠率与沙埋深度呈正相关。当沙 埋深度超过沙鞭幼苗能够出土的最大深度 $(6 \mathrm{~cm})$ 后, 部分种子的萌发被抑制。在 $6 \mathrm{~cm}$ 的深度, 有 $58 \%$ 的种子处于强迫休眠状态。导致植物种子的 强迫休眠的可能原因有: 土壤通气不良, 土壤含水量 过高, 土壤温度过低或过高, $\mathrm{O}_{2}$ 浓度低, $\mathrm{CO}_{2}$ 浓度高 以及光强不足等 (Maun, 1998; Huang \& Gutterman, 1998; Huang et al., 2004)。对于沙鞭来说, 土壤中 处于休眠状态的种子会形成长期种子库。种子库的 形成有利于物种的长期生存, 因为当沙子运动 (沙 蚀)减小沙埋深度时, 休眠的种子能够萌发出苗。这 种沙层移动的调节机制使土壤种子库里的种子在一 定时间只有部分种子萌发并出苗 (Gutterman, 2002), 与我们的野外调查结果一致。但是, 沙鞭种子在沙 埋之后中有一部分会死亡(约 $15 \% \sim 35 \%$ )。种子 在沙层里死亡的可能原因有真菌侵染 (Maun, 1998), 种皮里有水溶性的萌发抑制剂, 微生境不适 宜以及 $\mathrm{O}_{2}$ 含量太低等( Ren et al , , 2002)。

在沙埋条件下, 沙鞭种子大小的多样性也可能 影响其种子萌发和幼苗出土, 并且对该物种的种群 更新有一定的影响 ( van der Valk, 1974; Yanful \& Maun, 1996a)。这是因为种子的大小不同, 其传播 能力、萌发率、萌发速度以及萌发的时间可能不同。 这些种子形成的幼苗的大小、活力以及生存能力也 可能不同 (Baskin \& Baskin, 1998; Gutterman, 2002)。 与沙鞭相似, 很多植物都会产生大小不同的种子, 它 们的萌发力各不相同。一些植物的大种子的萌发率 比小种子的高 (Greipsson \& Davy, 1995; Yanful \& Maun, 1996a)。另一些植物的小种子的萌发率比大 种子的高 (Baskin \& Baskin, 1998)。但是某些植物的 种子虽然大小不同, 其萌发率却没有区别 (Chen \& Maun, 1999; Huang et al., 2004)。冰草属的 Agropyron psammophilum 的不同大小的种子的萌发率没有 区别, 但是大种子萌发的比较快 (Zhang \& Maun, 1990b）。我们的实验结果表明, 沙鞭的种子大小对 其在不同深度的沙层中的萌发率没有影响。很多因 素, 例如资源有限时种子之间的竞争、遗传因素以及 种子大小与种子数量的权衡, 都会使植物产生大小
不同的种子(Zhang \& Maun, 1990b)。

研究表明, 种子大小在决定幼苗能够出土的深 度时会起重要作用。对于某个特定的物种来说, 大 种子能够出土的土壤深度比小种子大 (Baskin $\&$ Baskin, 1998; Maun \& Payne, 1989)。例如 Cackie edentula 的果序上部的种子的平均质量为 (6.30 \pm $0.14) \mathrm{mg}$, 其幼苗能够出土的最大深度为 $12 \mathrm{~cm}$; 果 序下部的种子的平均质量为 $(4.70 \pm 0.13) \mathrm{mg}$, 其 幼苗能够出土的最大深度仅为 $8 \mathrm{~cm}$ (Maun \& Lapierre, 1986)。柠条( Caragana microphylla) 和羊柴 (Hedysarum laeve) 的幼苗出土率与其种子大小成正 相关 (Zhu et al., 2004)。很多物种的大种子形成的 幼苗比小种子形成的幼苗大, 因为大种子比小种子 贮存了更多的能量, 可以供幼苗早期生长使用。因 此, 大种子形成的幼苗有更强的生长势, 抵抗不良环 境条件的能力更强, 所以生存率更高 (Zhang, 1996; Baskin \& Baskin, 1998)。例如, 在实验室里培养时, Agropyron psammophilum 的大种子形成的幼苗在 $10 \sim$ $20 \mathrm{~d}$ 之内的叶面积和生物量比小种子形成的幼苗大 （Zhang \& Maun, 1990a)。在黑暗中培养时, 赖草属 的 Leymus arenarius 的幼苗的叶子长度随着种子质量 的增加而延长, 这种延长生长可以预测它们在沙埋 时能否出土 (Greipsson \& Davy, 1996)。Strophostyles helvola 的大种子在深层沙埋下的出苗率和幼苗存活 率都比小种子高, 其种子质量的高度变异是一种适 应, 因为大种子形成的幼苗不仅能够从更深的沙埋 中出土, 而且在沙埋条件下生存的机会更大 (Yanful \& Maun, 1996b)。此外, 在不同物种之间, 例如在 Ammorphila breviligulata, Corispermum edentula, $C$. hysopifolium 和 Eleymus canadensis 这 4 种植物中, 种 子大的物种的 ED50 (幼苗出土率达到 50\% 的深度, $\mathrm{cm}$ ) 和 Edmax (幼苗能够出土的最大深度, $\mathrm{cm}$ ) 较大 (Maun \& Lapierre, 1986)。Zhang 和 Maun(1990b)认 为, 在种子萌发之后, 冰草属植物 Agopyron psammophilium 的幼苗能否出土主要是由胚乳中剩余的 拒藏物质的量决定的。由于大种子里含有的胚乳较 多, 储藏了更多的能量, 当它们处于较深的土壤中 时, 就有足够的能量形成更长的茎, 可以保证幼苗出 土(Zhu et al ., 2004)。和小种子相比, 新疆大赖草 (Leymus racemosus) 的大种子的优势在于, 在阶段性 干燥后, 它所形成的幼苗的恢复生长率较高 (Huang \& Gutterman, 2004）。我们认为, 尽管沙鞭的种子萌 发率与种子大小无关, 但是大种子形成的幼苗具有 较高的出苗机率。大种子含有较多的咜藏物质还有 
可能使产生的幼苗具有更长的茎, 从而增加幼苗定 居的机会。前人在对 Cirsium pitcheri 的种子质量与 幼苗生长的关系进行研究得出的结论 (Chen \& Maun，1999）支持了我们的观点。

由于所败藏的能量不同, 沙鞭不同大小的种子 能够在不同的沙埋深度出土。在流动沙丘生境里, 沙埋比较剧烈。沙鞭产生的大种子因为咜存足够的 能量而具有生态优势, 能够保证处于沙土之下一定 深度的种子萌发之后形成的幼苗能够及时出土, 有 利于幼苗定居, 从而促进种群的更新。因此, 沙埋可 能是对沙生植物的种子大小的一种选择力 (Maun, 1998)。在沙埋比较剧烈的流动沙丘生境中, 沙鞭的 种子正是由于具有大小的多样性, 增加萌发后的幼 苗在不同沙埋深度建成的机会, 提高它们适应这种 环境胁迫的能力, 从而减少了生存的风险。

\section{参 考 文 献}

Baskin CC, Baskin JM (1998). Seed Ecology, Biogeography, and Evolution of Dormancy and Germination. Academic Press, San Diego, 212 - 534 .

Chen H, Maun MA (1999). Effects of sand burial depth on seed germination and seedling emergence of Circium pitcheri. Plant Ecology, 140, 53-60.

Dannin A (1996). Plants of Desert Dunes. Springer-Verlag, New York, 3-19.

Dong M (董鸣) (1999). Effects of severing rhizome on clonal growth in rhizomatous grass species Psammochloa villosa and Leymus secalinus. Acta Botanica Sinica (植物学报), 41, 194 - 198. (in Chinese with English abstract)

Dong M, Alaton B (1999). Clonal plasticity in response to rhizome serving and heterogeneous resource supply in the rhizomatous grass Psammochloa villosa in an Inner Mongolia dune, China. Plant Ecology, 141, 53-58.

Gutterman Y (1993). Seed Germination in Desert Plants. Adaptation of Desert Organisms. Springer-Verlag, Berlin, 140 - 144, $222-230$.

Gutterman Y (2002). Survival Strategies of Annual Desert Plants. Adaptations of Desert Organisms. Springer-Verlag, Berlin, 211 280 .

Greipsson S, Davy AJ (1995). Seed mass and germination behavior in population of the dune-building grass Leymus arenarius. Annals of Botany, 76, $493-501$.

Greipsson S, Davy AJ (1996). Sand accretion and salinity as constraints on the establishment of Leymus arenarius for land reclamation. Annals of Botany, 78, 611-618.

Harris D, Davy AJ (1987). Seedling growth in Eleymus farctus after episodes of burial with sand. Annals of Botany, 60, 587 593.

Huang ZY (黄振英) (2003). Adaptation strategies of seed dor- mancy and germination of Psammochloa villosa, a sand dune grass inhabiting Ordos Plateau, China. Acta Botanica Boreali Occidentalia Sinica (西北植物学报), 23,1128-1138.（in Chinese with English abstract)

Huang ZY, Gutterman Y (1998). Artemisia monosperma achene germination in sand: effects of sand depth, sand/moisture content, cyanobacterial sand crust and tempreture. Journal of Arid Environments, 38, $27-43$.

Huang ZY (黄振英), Gutterman Y (2000). Comparison of germination strategies of Artemisia ordosica with its two congeners from deserts of China and Israel. Acta Botanica Sinica (植物学报), $42,71-80$.

Huang ZY, Gutterman Y (2004). Seedling desiccation tolerance of Leymus racemosus (Gramineae) (wild rye), a sand dune grass inhabiting the Junggar Basin of Xinjiang, China. Seed Science Research, 14, 233-239.

Huang ZY, Dong M, Gutterman Y (2004). Caryopses dormancy, germination and seedling emergence in sand, of Leymus raemosus (Poaceae), a perennial sand dune grass inhabiting the Junggar Basin of Xinjiang, China. Australian Journal of Botany, 52, 519 -528 .

Huang ZY (黄振英), Gutterman Y, Hu ZH (胡正海), Zhang XS (张新时) (2001). Seed germination in Artemisia sphaerocephala II . The influence of environmental factors. Acta Phytoecologica Sinica (植物生态学报), 25,240-246. (in Chinese with English abstract)

International Seed Testing Association (1985). International rules for seed testing. Seed Science and Technology, 13, 299-355.

Liu YX (刘瑛心) (1985). Flora in Desertis Reipublicae Populorum Sinarum. Vol. I (中国沙漠植物志). Science Press, Beijing, 105. (in Chinese)

Ma YQ (马毓泉) (1998). Flora Intramongolica. Tomus 5 (内蒙 古植物志). Inner Mongolia People Press, Huhhot, 219. (in Chinese)

Maun MA (1981). Seed germination and seedling establishment of Calamovilfa longifolia on Lake Huron sand dunes. Canadian Journal of Botany, 59, 460-469.

Maun MA (1998). Adaptations of plants to burial in coastal sand dunes. Canadian Journal of Botany, 76, 713- 738 .

Maun MA, Lapierre J (1984). The effects of burial by sand on Ammophila breviligulata. Journal of Ecology, 72, 827 - 839 .

Maun MA, Lapierre J (1986). Effects of burial by sand on seed germination and seedling emergence of four dune species. American Journal of Botany, 73, 450-455.

Maun MA, Payne AM (1989). Fruit and seed polymorphism and its relation to seedling growth in the genus Cakile. Canadian Journal of Botany, 67, $2743-2750$.

Ren J, Tao L, Liu XM (2002) . Effect of sand burial depth on seed germination and seedling emergence of Calligonum L. Species. Journal of Arid Environments, 51, 603-611.

Roles B, Donders S, Werger M, Dong M (2001). Relation of 
wind-induced sand displacement to plant biomass and plant sandbinding capacity. Acta Botanica Sinica (植物学报), 43, 979 -982 .

Sokal RR, Rohlf EJ (1981) . Biometry. Freeman, San Francisco, CA, 859.

Sykes MT, Wilson JB (1990). An experimental investigation into response of New Zealand sand dune species to different depths of burial by sand. Acta Botanica Neerlandica, 39, $171-181$.

van Assche JA, Vanlerberghe VA (1989). The role of temperature on dormancy cycle of seeds of Rumex obtusifolia L. Functional Ecology, 3, 107 - 115 .

van der Valk AG (1974). Environment factors controlling the distribution of forbs on coastal foredunes in Cape Hatteras National Seashore. Canadian Journal of Botany, 52, 1057 - 1073 .

Vleeshouwers LM (1997). Modeling the effect of temperature, soil penetration resistance, burial depth and seed weight on pre-emergence growth of weeds. Annals of Botany, 79, 553- 563 .

Yanful M, Maun MA (1996a). Spatial distribution and seed mass variation of Strophostyles helvola along Lake Erie. Canadian Journal of Botany, 74, 1313 - 1321 .

Yanful M, Maun MA (1996b). Effects of burial of seeds and seedlings from different seed size on the emergence and growth of Strophostyles helvola. Canadian Journal of Botany, 74, 1322 1330 .

Zhang CY (张称意), $\mathrm{Yu} F H$ (于飞海), Dong M (董鸣) (2002). Effects of sand burial on the survival, growth and biomass allocation in semi-shrub Hedysarum laeve seedlings. Acta Botanica Sinica (植物学报), 44, 337 - 343 .

Zhang JH, Maun MA (1990a). Seed size variation and its effects on seedling growth in Agropyron psammophilum. Botanical Gazette, 151, $106-113$.

Zhang JH, Maun MA (1990b). Sand burial effects on seed germination, seedling emergence and establishment of Panicum virgatum. Holarctic Ecology, 13, 56-61.

Zhang JH, Maun MA (1990c). Effects of sand burial on seed germination, seedling emergence, survival, and growth of Agropyron psammophilum. Canadian Journal of Botany, 68, 304-310.

Zhu XW, Huang ZY, Liu HD, Chu Y, Zhang SM, Dong M (2004). Effects of burial in sand and seed size on seed germination and seedling emergence in two leguminous shrubs in the Otindag Sandland, China. Israel Journal of Plant Sciences, 52, $133-142$. 